\title{
NON THERMAL SPUTTERING OF GRAINS AND PRODUCTION OF SIO IN INTERSTELLAR SHOCKS
}

\author{
GUILLAUME PINEAU DES FORÊTS \\ DAEC, Observatoire de Paris \\ 92195 Meudon Cedex, France \\ AND \\ DAVID FLOWER \\ Physics Department, The University \\ Durham DH1 3LE, UK
}

\begin{abstract}
We present recent results for the yields of Si and O, produced in the sputtering of $\mathrm{SiO}_{2}$ by ions of different masses, and show the importance of sputtering by heavy particles at low streaming velocities. These data are incorporated in a C-shock model to study the erosion of interstellar grains and the release of silicon through non-thermal sputtering within the shock. Once in the gas phase, the atomic silicon reacts with $\mathrm{O}_{2}$ and is rapidly transformed into $\mathrm{SiO}$. The column densities of $\mathrm{SiO}$ thus calculated are compared with the observations of molecular outflows with a satisfactory agreement. In the postshock gas, $\mathrm{SiO}$ disappears from the gas phase through the reaction $\mathrm{SiO}(\mathrm{OH}, \mathrm{H}) \mathrm{SiO}_{2}$ and $\mathrm{SiO}_{2}$ remains, unseen, in the cold dense gas. This could explain the extremely low upper limits of $\mathrm{SiO}$ deduced from observations of dark clouds.
\end{abstract}

\section{Introduction}

One of the main conclusions from recent observations of the interstellar medium is that molecular clouds seem to exhibit strong variations in their molecular abundances; this is the case from cloud to cloud, and also within the same cloud. These variations are usually explained as effects of various physical mechanisms which relate to the cloud environment or structure (clumpiness, shocks, collapse, radiation field, turbulence), requiring sophisticated theoretical models. It is more difficult to deduce variations 
in elemental abundances from observations (see the review by Meyer in this volume). However, it is clear that heavy elements such as silicon exhibit strong variations in their degree of depletion. Dust-grain cores are formed in stellar atmospheres (and probably also in molecular clouds) and are ejected in stellar outflows into the interstellar medium, where they may grow through the accretion of interstellar molecules, which form mantles on their surfaces. Heavy elements such as $\mathrm{Si}, \mathrm{Mg}, \mathrm{Fe}, \mathrm{Ca}$ and $\mathrm{P}$ are locked up in refractory materials; this explains the depletions with respect to cosmic abundances that are observed in both diffuse (Sofia, Cardelli \& Savage 1994) and in dark clouds, where the degree of depletion is even greater (see the review by Turner 1991).

Shock waves propagating in the interstellar medium are expected to erode and partly destroy the dust through sputtering and grain-grain collisions. The observed elemental depletions are believed to be the result of a balance between the injection of "stardust" and grain growth and destruction in the interstellar medium. However, the grain lifetime estimated from the destruction processes in shocks is considerably shorter than the timescale for recycling the interstellar material through stars (McKee 1989, Draine 1990). For this reason alone, it is important to establish as accurately as possible the rate at which the refractory materials are destroyed in shock waves.

In this paper, we consider the formation of silicon-bearing species in a molecular cloud in which a shock wave is propagating. Large enhancements of the gas-phase silicon abundance are observed in high velocity components, not only of diffuse clouds (Sofia, Savage \& Cardelli 1993, Gry et al. 1997), but also of dense clouds associated with supernova remnants (van Dishoeck, Jansen \& Phillips 1993). In molecular outflows, up to $3 \%$ of the silicon can be in the gas phase, in the form of $\mathrm{SiO}$ (Martín-Pintado, Bachiller \& Fuente 1992). The spatial and velocity distributions of the SiO emission suggest that $\mathrm{SiO}$ is formed in shocked layers, in which grains have been partially destroyed, releasing silicon into the gas phase.

Molecular outflows are the result of the interaction between a high velocity jet from a young star and the surrounding molecular environment, from which the star originally formed. The supersonic speeds generate confined mixing layers and bow shocks which can be considered, in a first approximation, to be a sum of shocks of different types and velocities distributed along the interface between the jet and the ambient molecular medium. A detailed model of such regions would require the complete numerical resolution of a 3-D (magneto)hydrodynamical problem, incorporating all the physical and chemical processes involved. Such a problem is probably not soluble with the computers which are currently available. Hydrodynamical models, with a simplified treatment of the chemistry and cooling processes, 
have been constructed, for example, by Raga, Cabrit \& Cantó (1995) and are discussed in this volume by Raga. However, bow shocks have probably C-shock characteristics in their wake (Smith 1991). Our approach, although limited to one dimension, has the merit of following simultaneously not only the dynamics but also the gas-phase chemistry and the interactions with the grains.

A brief review of the observed silicon abundances observed in dense clouds is given in Section 2. The non-thermal sputtering processes occurring in shocks are summarized in Section 3. In Section 4 we present recent results for sputtering yields at low energies, and in Section 5 we illustrate the erosion of dust grains by means of a representative C-shock model. The silicon chemistry and the mechanisms of formation and destruction of $\mathrm{SiO}$ in the shock are presented in Section 6. In Section 7, we demonstrate the importance of sputtering by heavy particles through a comparison of models. Our concluding remarks are given in Section 8.

\section{Silicon in dense clouds}

In dark clouds, most of the gas-phase silicon is predicted to be in the form of $\mathrm{SiO}$, formed in the reaction $\mathrm{Si}\left(\mathrm{O}_{2}, \mathrm{O}\right) \mathrm{SiO}$ (Herbst et al. 1989). However, $\mathrm{SiO}$ is not observed in the quiescent components of dense clouds, and extremely low upper limits have been deduced from observations. For example, in TMC-1 Ziurys, Friberg \& Irvine (1989) found that $n(\mathrm{SiO}) / n\left(\mathrm{H}_{2}\right)<310^{-12}$, which implies that the fraction of silicon in the gas phase is less than $10^{-7}$. Most of the silicon in dense clouds must be tied-up in silicates. Moreover, $\mathrm{SiO}$ has never been detected in photodissociation regions (Jansen et al. 1995).

On the other hand, observations of molecular outflows show the abundance of gas-phase $\mathrm{SiO}$ to be several orders of magnitude larger than in dark clouds. The $\mathrm{SiO}$ emission is kinematically well displaced from the bulk cloud velocity and probably arises in shocked material. For example, in the $\mathrm{L} 1448$ outflow, the $\mathrm{SiO} / \mathrm{H}_{2}$ abundance ratio is estimated to be a few $10^{-7}$ in the high velocity gas (implying a fractional abundance of Si comparable to that in diffuse clouds) but less than $10^{-11}$ in the ambient medium (Bachiller, Martín-Pintado \& Fuente 1991). The most likely explanation is that the silicon is released from the grains by the action of shocks, which are associated with bipolar outflows, in which case $\mathrm{SiO}$ emission is a good tracer of shock activity.

\section{Non-thermal sputtering by shocks}

Kinetic temperatures as high as $10^{5} \mathrm{~K}$ would be necessary for thermal sputtering to occur, and such temperatures can be reached only in fast, non- 
radiative shocks (Seab 1987). In fact, non-thermal sputtering dominates the process of grain erosion in the interstellar medium (see Draine 1995 for a review of grain destruction in interstellar shocks). In regions where the fractional ionization is high, the neutrals, ions and the magnetic field are well coupled and shocks propagate as single-fluid structures in which there is a discontinuity ( $\mathrm{J}$-shocks). The magnetic field is compressed in the cooling gas behind the shock front, and charged grains accelerate around the field lines, producing gas-grain velocities which can be comparable to the shock velocity (Spitzer 1976). This "betatron acceleration" appears to be the main mechanism of grain destruction in the diffuse interstellar medium for shock velocities in the range $50-200 \mathrm{~km} \mathrm{~s}^{-1}$ (Draine \& Salpeter 1979b, Seab \& Shull 1983, McKee et al. 1987, Jones et al. 1994).

In dark clouds, where the fractional ionization is low, the coupling between neutral and charged particles is weak and shock propagation results in a multifluid structure. Charged particles are coupled to the magnetic field and are heated and accelerated ahead of the neutral fluid (Mullan 1971, Draine 1980). The heating and acceleration of the neutrals occurs through collisions with ions and charged grains, and all the flow variables can vary continuously (C-shocks). Large streaming velocities can be reached between the ions and the neutrals, and charged dust grains move through the neutrals with a drift velocity close to the ion-neutral velocity (Havnes, Hartquist \& Pillip 1987). Non-thermal sputtering of the grains then occurs in collisions with the neutrals within the shock (Draine, Roberge \& Dalgarno 1983, Flower \& Pineau des Forêts 1995).

Grain-grain collisions in interstellar shocks have been studied by Jones et al. $(1994,1996)$. This process seems to be sufficient to break large grains into smaller ones and hence to modify the grain-size distribution; it could also be responsible for returning silicon to the gas phase, through vaporization, when the relatives speeds of the grains exceed a few $\mathrm{km} \mathrm{s}^{-1}$.

\section{Sputtering yields of the refractory grains}

Numerous measurements of sputtering yields have been made in the laboratory for ion impact on the surfaces of various materials (see the reviews by Andersen \& Bay 1981 and by Betz \& Wehner 1983). However, experiments at low energies are difficult to perform and sputtering yields have generally been measured at impact energies higher than $100 \mathrm{eV}$. Furthermore, there are significant discrepancies between different sets of measurements, and the only materials of astrophysical interest that have been studied are amorphous carbon and $\mathrm{SiO}_{2}$. Unfortunately, no measurements have been made on olivine $\left((\mathrm{Mg}, \mathrm{Fe}) \mathrm{SiO}_{4}\right)$, which is believed to be one of the main forms of refractory dust in the interstellar medium. Prescriptions for ex- 
trapolating the sputtering yields towards lower energies have been given by Barlow (1978), Draine \& Salpeter (1979a) and Tielens et al. (1994), who considered only $\mathrm{H}^{+}$and $\mathrm{He}^{+}$as impacting particles. These extrapolations broadly agree in predicting a rapid drop in the sputtering yields below 100 $\mathrm{eV}$ and indicate that the yield for $\mathrm{He}^{+}$impact on $\mathrm{SiO}_{2}$ attains $210^{-4}$ at an energy of about $30 \mathrm{eV}$ (see Fig. 1). However, until recently, no attempt has been made to compute numerically the sputtering yields in the domain of energies below $100 \mathrm{eV}$ which is relevant to many astrophysical applications (and which corresponds to a velocity $<70 \mathrm{~km} \mathrm{~s}^{-1}$ for $\mathrm{He}^{+}$impact).

Field et al. (1996) report calculations of the sputtering yields of Si and $\mathrm{O}$, produced by ion impact on $\mathrm{SiO}_{2}$, and of $\mathrm{C}$ from amorphous carbon. The yields were calculated using the classical trajectory simulation code TRIM (TRansport of Ions in Matter), developed for the theoretical study of the ion bombardment of surfaces (Ziegler, Biersack \& Littmark 1985), which has been extensively used in the electronic device and nuclear industries. No distinction needs to be made between ion and neutral impact, as ions capture electrons as they approach the solid surface. TRIM follows the trajectory of the incident particle as it penetrates the solid and undergoes a series of binary collisions, depositing energy in the material and perhaps ejecting one or more atoms of which the material is composed, until it exits the surface (as a back-scattered atom) or becomes implanted. The target atoms are also followed through subsequent collisions, until they lose so much energy that they can no longer displace other atoms, or they pass through the surface and leave the target as sputtered atoms; $10^{6}$ trajectories were followed for each energy and angle of incidence. The probability of ejection was deduced as the ratio of the number of sputtered atoms to the total number of impacting particles, at a given angle and energy, and then averaged over the angles to produce the sputtering yield as a function of the energy. The principal sources of uncertainty are: (i) the lack of accurate knowledge of the displacement and binding energies; (ii) the approximate representation of the interaction potential (only binary collisions are included); and (iii) the neglect of prior implantation and consequent damage to the target. Details of the method can be found in Field et al. (1996), where the fits of the sputterings yields to the impact energy are also given.

Fig. 1 shows the combined sputtering yields of $\mathrm{Si}$ and $\mathrm{O}$ calculated for $\mathrm{He}^{+}$impact on $\mathrm{SiO}_{2}$. At high energies, the results derived using TRIM (thick line) are in rather good agreement with the available measurements of Roth, Bohdansky \& Ottenberger (1979). At low energies the impact energy at which the onset of sputtering occurs is slightly higher than predicted by the extrapolation formulae: the yield attains $210^{-4}$ at an energy of about $37 \mathrm{eV}$ instead of $30 \mathrm{eV}$ deduced by Tielens et al. (1994). As we are interested in the formation of $\mathrm{SiO}$, the sputtering yield of $\mathrm{Si}$ is also shown 


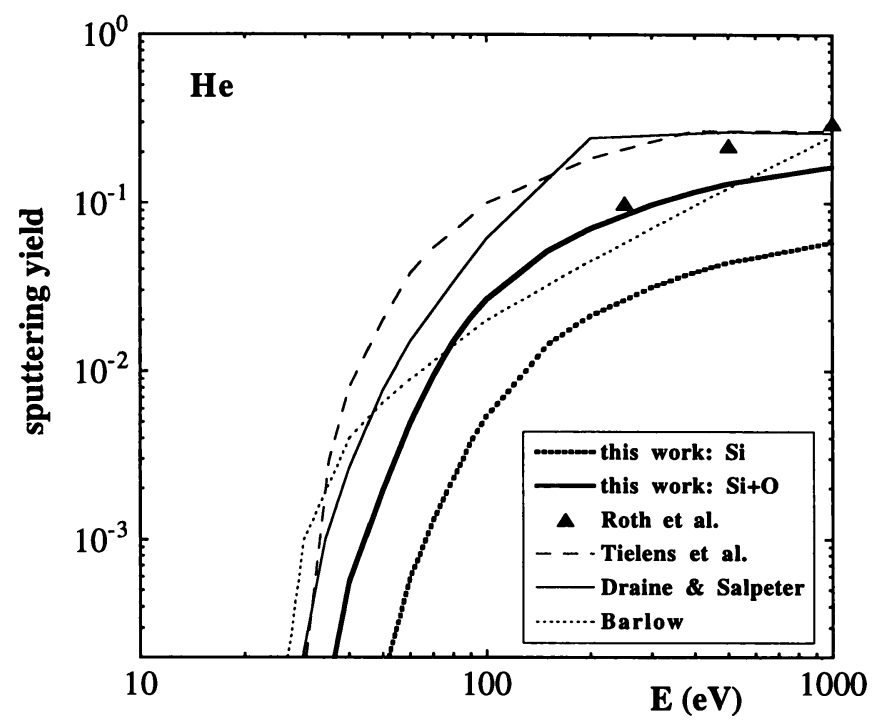

Figure 1. Angle-averaged sputtering yields for the impact of $\mathrm{He}^{+}$on amorphous $\mathrm{SiO}_{2}$ as a function of the energy of the incident ion. The heavy solid line represents the combined yield of $\mathrm{Si}$ and $\mathrm{O}$, and the heavy dotted line the calculated yield for the release of $\mathrm{Si}$ alone. Also shown are the experimental data of Roth et al. (1979) and the different extrapolations towards low energies proposed by Barlow (1978), Draine \& Salpeter (1979a) and Tielens et al. (1994).

in Fig. 1 (thick dotted line). As expected, sputtering of Si begins at much higher impact energies than the sputtering of $O$ because of its larger mass. One can immediately deduce from these results that only "high velocity" shocks (leading to streaming velocities greater than $40 \mathrm{~km} \mathrm{~s}^{-1}$ ) will release significant amounts of silicon into the gas phase through He impact.

At low streaming velocities, sputtering by species heavier than He needs to be considered. Fig. 2 shows the sputtering yield for the release of Si from amorphous $\mathrm{SiO}_{2}$, using $\mathrm{C}, \mathrm{O}, \mathrm{Si}$ and $\mathrm{Fe}$ ions as the impacting particles, plotted as a function of the velocity of the incident particle. The velocity at which significant sputtering occurs decreases with increasing mass of the incident particle because of the greater kinetic energy at a given velocity. The sputtering yield for He impact is also shown for comparison. For low velocities $\left(<40 \mathrm{~km} \mathrm{~s}^{-1}\right)$, the yield for sputtering by He is lower by many orders of magnitude than for heavy particles. Consequently, in "low velocity" shocks, sputtering by heavy particles dominates in spite of their much lower abundance in the interstellar medium $\left(\approx 10^{-3}\right.$ relative to $\left.\mathrm{He}\right)$, as will be shown below. 


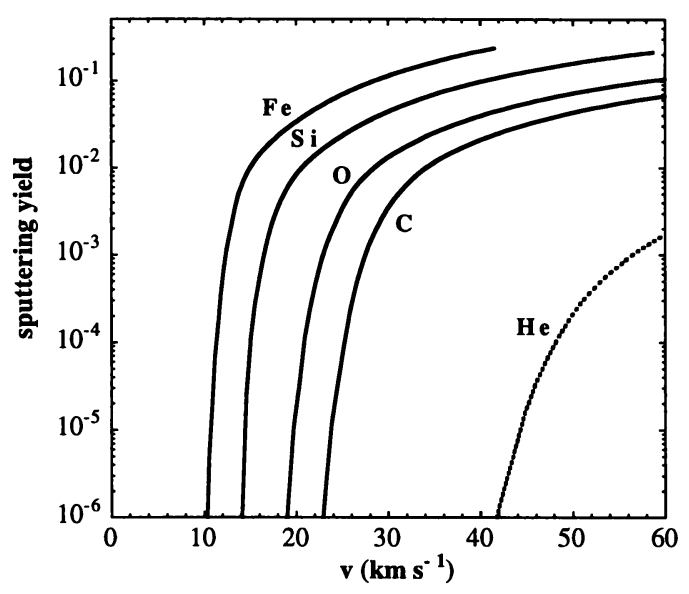

Figure 2. Calculated sputtering yields for the impact of $\mathrm{C}, \mathrm{O}, \mathrm{Si}$ and $\mathrm{Fe}$ on amorphous $\mathrm{SiO}_{2}$ (with $\mathrm{Si}$ as the sputtered product) as a function of the velocity of the incident particle. The sputtering yield for He impact is also shown for comparison.

\section{The shock model}

Recently, Schilke et al. (1997) have studied the production of SiO in the gas phase of molecular outflows, following the sputtering of Si from amorphous $\mathrm{SiO}_{2}$. To illustrate this process, we shall concentrate on a representative model of a C-shock which propagates at a velocity of $25 \mathrm{~km} \mathrm{~s}^{-1}$ in a dark cloud with a preshock density of $10^{5} \mathrm{~cm}^{-3}$ and a magnetic field of 200 $\mu \mathrm{G}$ (supposed transverse to the direction of propagation of the shock). The temperature and density profiles are plotted in Fig. 3, which shows the strong decoupling of the temperatures of the ions and neutrals owing to the low degree of ionization of the gas. The ions (and electrons) are heated prior to the neutrals and their temperature reaches a maximum of almost $410^{4} \mathrm{~K}$, while the temperature of the neutrals attains only $2000 \mathrm{~K}$ within the shock. The postshock density is $310^{6} \mathrm{~cm}^{-3}$, corresponding to a compression factor of 30 .

Fig. 4 shows the ion and neutral velocities $\left(v_{i}\right.$ and $\left.v_{n}\right)$ expressed in the shock frame (i.e. the preshock gas is on the left in this Fig.). Again, the strong decoupling of the neutral and charged particles is apparent. The ion velocity falls first (in this frame), resulting in deceleration of the neutrals through ion-neutral collisions until, in the postshock gas, the ion and neutral velocities recouple. The rate of momentum transfer between the ionized and neutral fluids regulates the shock profile (Flower \& Pineau des Forêts 1995). Within the shock, sputtering occurs through neutral particle impact on charged grains at the speed $\Delta v=v_{n}-v_{i}$ (also plotted in Fig. 4). 


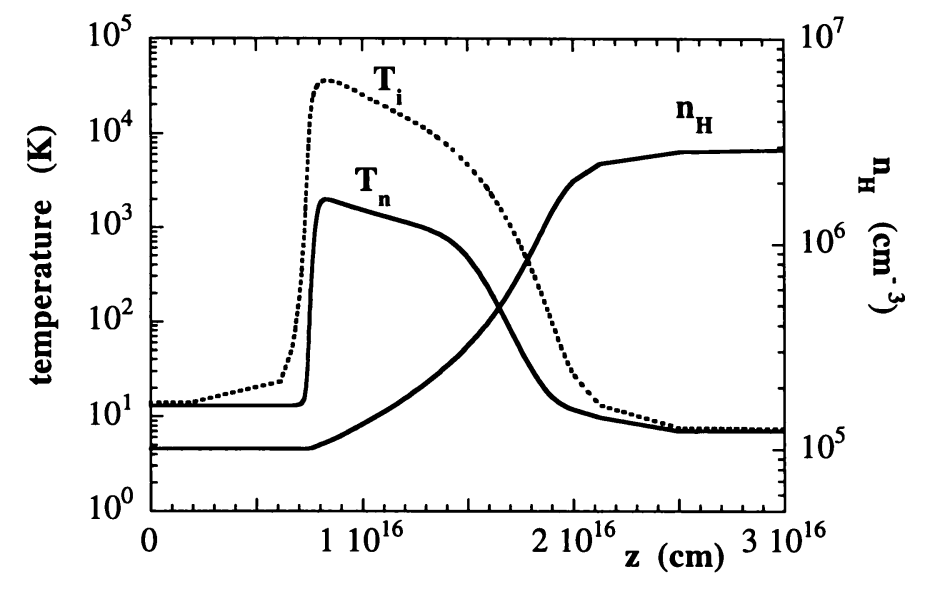

Figure 3. Number density $\left(\mathrm{n}_{H}=\mathrm{n}(\mathrm{H})+2 \mathrm{n}\left(\mathrm{H}_{2}\right)\right)$ and temperature profiles of the neutrals $\left(\mathrm{T}_{n}\right)$ and ions $\left(\mathrm{T}_{i}\right)$ in the C-shock described in Section 5 , plotted as a function of the distance $\mathrm{z}$ behind the shock front.

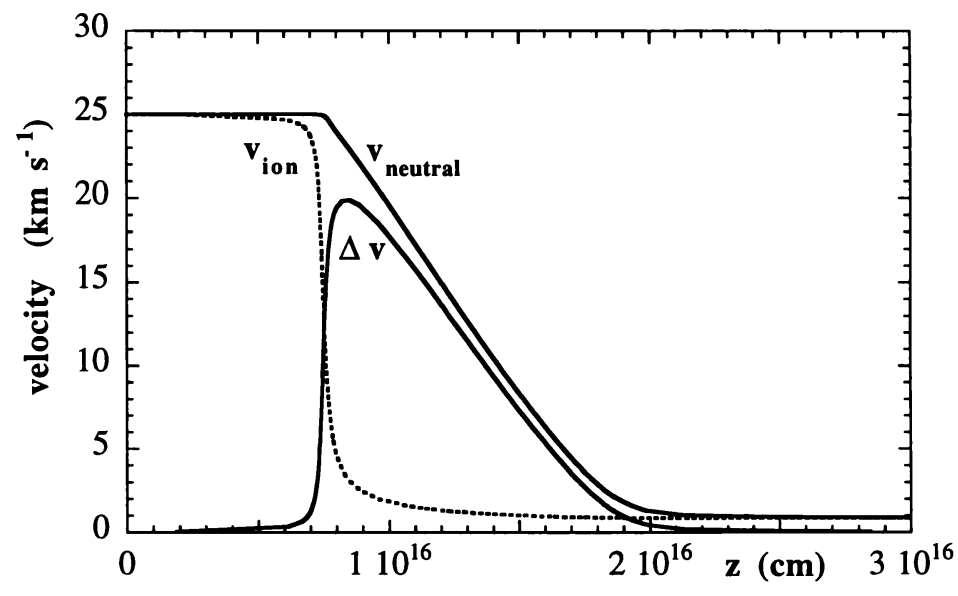

Figure 4. Velocity profiles of the neutral particles (full line) and of the ions (dotted line) in the reference frame of the shock; the ions and the neutrals flow into the shock from the left at the shock speed. The ion-neutral velocity difference $\Delta v=v_{n}-v_{i}$ is also plotted. All the velocities are expressed in $\mathrm{km} \mathrm{s}^{-1}$.

This drift velocity attains a maximum of $20 \mathrm{~km} \mathrm{~s}^{-1}$, close to the shock velocity. Each grain collides with many times its own mass of gas within the shock.

Grains with the size distribution deduced by Mathis, Rumpl \& Nord- 
sieck (1977) and the sputtering data described in Section 4 have been incorporated in the MHD code published by Heck, Flower \& Pineau des Forêts (1990). The gas-phase and grain-surface chemistries have also been included. The "heavy particles" mentioned in Section 4 have been identified with the most abundant species in the shock $\left(\mathrm{C}, \mathrm{N}, \mathrm{O}, \mathrm{CO}, \mathrm{N}_{2}, \mathrm{O}_{2}\right.$ and $\mathrm{H}_{2} \mathrm{O}$ ) with an associated sputtering yield equal to that calculated for the impacting particle of nearest mass. Further information on this code and on the chemistry included may be found in Schilke et al. (1997). In the preshock gas, the silicon is supposed to be entirely confined to the silicate grain cores, represented by amorphous $\mathrm{SiO}_{2}$, and we restrict the discussion here to the process of grain core erosion. Mantle erosion has been studied by Flower \& Pineau des Forêts (1994), Flower, Pineau des Forêts \& Walmsley (1995) and Schilke et al. (1997). The fractional abundance of elemental silicon was taken to be $3.610^{-5}$ relative to hydrogen (Anders \& Grevesse 1989). The other elemental abundances and their initial distribution among the different phases of the model can be found in Table 2 of Flower et al. (1995).

\section{Silicon chemistry and formation of $\mathrm{SiO}$}

As we have already seen, the amount of silicon released at low energies depends strongly on the shock velocity because of the steep slope of the sputtering yield near threshold. In the representative model presented here, approximately $3 \%$ of the silicon is returned to the gas phase, as can be seen in Fig. 5.

In the shock, the silicon chemistry is driven mainly by neutral-neutral reactions, and $\mathrm{Si}$ is rapidly oxidized and transformed to $\mathrm{SiO}$ through the reactions

$$
\begin{aligned}
& \mathrm{Si}+\mathrm{O}_{2} \rightarrow \mathrm{SiO}+\mathrm{O} \\
& \mathrm{Si}+\mathrm{OH} \rightarrow \mathrm{SiO}+\mathrm{H},
\end{aligned}
$$

whose rate coefficients have been taken from Langer \& Glassgold (1990). Almost all the oxygen available (i.e. which is not locked in $\mathrm{CO}$ ) is transformed into water through the two successive reactions with activation barriers,

$$
\begin{gathered}
\mathrm{O}+\mathrm{H}_{2} \rightarrow \mathrm{OH}+\mathrm{H}-2980 \mathrm{~K} \\
\mathrm{OH}+\mathrm{H}_{2} \rightarrow \mathrm{H}_{2} \mathrm{O}+\mathrm{H}-1490 \mathrm{~K},
\end{gathered}
$$

and $\mathrm{H}_{2} \mathrm{O}$ becomes the most abundant oxygen-bearing molecule within the shock. When the gas cools, $\mathrm{H}_{2} \mathrm{O}$ is gradually transformed into $\mathrm{OH}$ through reactions with ions, and $\mathrm{OH}$ becomes abundant in the postshock gas before being dissociated itself in reactions with ions. Consequently, in the 


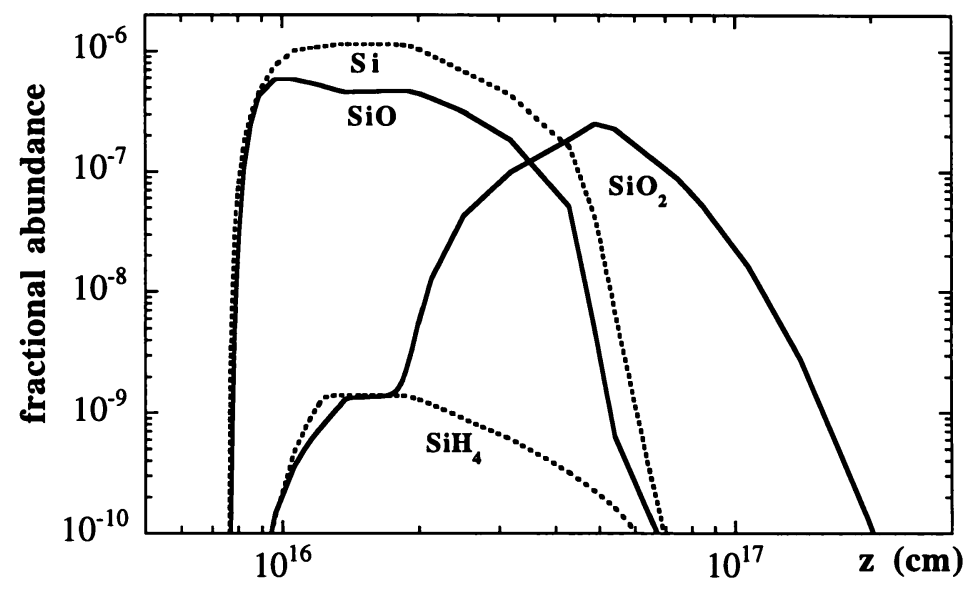

Figure 5. The fractional abundances, $\mathrm{n} / \mathrm{n}_{H}$, of selected silicon-bearing species in the gas phase, as functions of distance through the shock for the same model as in Figs. 2 and $4 . \mathrm{Si}$ is released from grains through sputtering and is transformed to $\mathrm{SiO}$ and $\mathrm{SiO}_{2}$ which are then adsorbed on grain surfaces in the cold postshock gas, disappearing from the gas phase.

postshock gas, $\mathrm{SiO}$ reacts with $\mathrm{OH}$ and is converted to $\mathrm{SiO}_{2}$ through the reaction

$$
\mathrm{SiO}+\mathrm{OH} \rightarrow \mathrm{SiO}_{2}+\mathrm{H}
$$

which may have a small activation barrier, as discussed by Langer \& Glassgold (1990). The complete list of the reactions involving silicon-bearing species, and their rate coefficients, can be found in Table 2 of Schilke et al. (1997).

Fig. 5 shows that $\mathrm{SiO}$ traces grain destruction in the shock. The thickness of the shocked layer is in agreement with the upper limit of $10^{-2} \mathrm{pc}$ derived by Martín-Pintado et al. (1992). The column density ratio SiO/CO attains $410^{-3}$, a value comparable to the estimations made by Bachiller et al. (1991) from observations of L1448. More hazardous is the direct comparison of the column density calculated using a plane-parallel model with that deduced from observations, because of geometrical effects and also the difficulty of determining the contribution of the cold postshock component from the observations. The column density of $\mathrm{SiO}$ in the hot shocked gas attains almost $10^{15} \mathrm{~cm}^{-2}$, which is higher than that deduced from observations of $\mathrm{SiO}$ in molecular outflows, which are in the range $10^{13}-10^{14}$ $\mathrm{cm}^{-2}$ (Martín-Pintado et al. 1992, Zhang et al. 1995; see also the article by Bachiller in this volume). However, the calculated column density depends strongly on certain physical parameters of the model, and, for example, 
would be less for lower shock velocities or higher preshock magnetic fields. Indeed, a lower $\mathrm{SiO}$ abundance is observed at smaller velocities in L1448 (Martín-Pintado et al. 1992). Most of the $\mathrm{SiO}$ is produced at the back of the shock, where the cooling neutral gas is being compressed. Consequently, the peak velocity of $\mathrm{SiO}$ is very close to the postshock velocity, which may explain why $\mathrm{SiO}$ is observed at velocities and positions different from those of the ambient medium.

A more complete comparison of models with observations has been made by Schilke et al. (1997), who solved for the excitation of $\mathrm{SiO}$ in the hot gas and calculated the $\mathrm{SiO}$ line intensities and profiles. They found satisfactory agreement between the calculations and observations. However, it remains difficult to deduce the shock velocities from the observations because of projection effects and the possibility that the shock runs not into the ambient gas, but into pre-accelerated gas.

Fig. 5 shows that, far behind the shock, the Si-bearing species are readsorbed on to the grains, disappearing from the gas phase (a sticking probability of unity was assumed in the cold postshock gas for all species). If adsorption processes are neglected, $\mathrm{SiO}_{2}$ (which is a stable molecule) is practically the only Si-bearing species in the postshock gas for a time as long as $10^{6} \mathrm{yr}$. It is eventually destroyed in reactions with atomic ions, and $\mathrm{SiO}$ becomes the main silicon bearing species, as predicted by steady-state models of the gas-phase chemistry in dark clouds (Herbst et al. 1989). SiO may have a very low abundance in the postshock gas, which could explain why it appears to be absent from the gas phase of dark clouds (Ziurys et al. 1989) and from photon-dominated regions such as the Orion Bar (Jansen et al. 1995).

\section{Importance of sputtering by heavy species}

To illustrate the importance of sputtering by heavy species, we compare in Fig. 6 the computed values of the streaming velocity, $\Delta v$, and the column density of $\mathrm{SiO}$ for two $\mathrm{C}$-shock models with the same values of the initial magnetic field strength $(B=200 \mu \mathrm{G})$ and of the preshock density $\left(n_{H}=10^{5} \mathrm{~cm}^{-3}\right)$. Fig. 6 shows that, if sputtering due to He impacts only is taken into account, shock velocities as high as $50 \mathrm{~km} \mathrm{~s}^{-1}(\Delta v \approx 43 \mathrm{~km}$ $\mathrm{s}^{-1}$ ) are needed to obtain column densities $N(\mathrm{SiO}) \approx 10^{14} \mathrm{~cm}^{-2}$, which are observed towards L1157 (Zhang et al. 1995). If sputtering by heavy species (mentioned in Section 5) is also included, shock velocities of about $20 \mathrm{~km}$ $\mathrm{s}^{-1}\left(\Delta v \approx 15 \mathrm{~km} \mathrm{~s}^{-1}\right)$ are sufficient to yield comparable column densities of SiO. We conclude that, in the interstellar medium, sputtering of amorphous $\mathrm{SiO}_{2}$ and carbon occurs only for streaming velocities $\Delta v>10 \mathrm{~km}$ $\mathrm{s}^{-1}$. In the range $10<\Delta v<50 \mathrm{~km} \mathrm{~s}^{-1}$, sputtering is dominated by heavy 


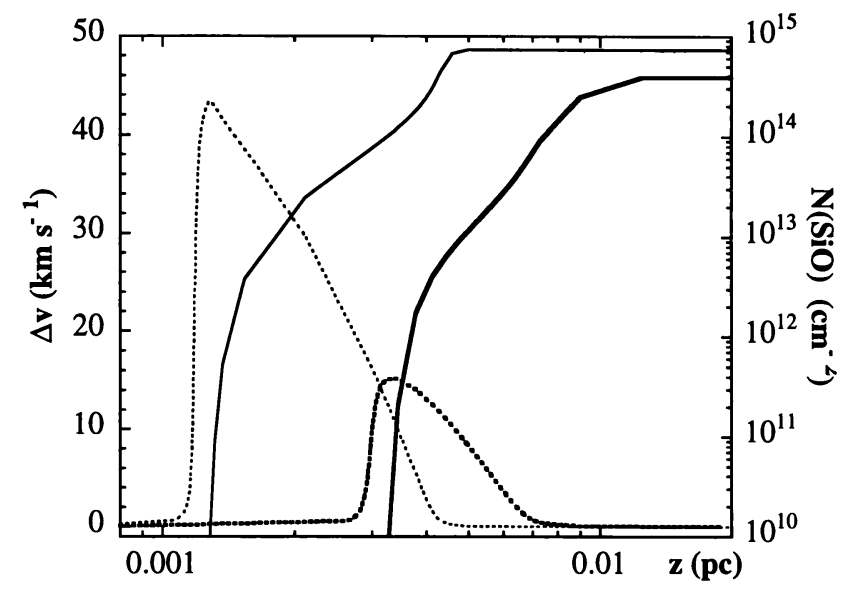

Figure 6. The ion-neutral velocity difference (dotted lines) and $\mathrm{SiO}$ column density (full lines) for two C-shock models. The light curves represent a model with a shock speed of $50 \mathrm{~km} \mathrm{~s}^{-1}$ where sputtering by helium only is taken into account. The heavy curves were obtained at a shock speed of $20 \mathrm{~km} \mathrm{~s}^{-1}$ on including the sputtering by "heavy particles".

particle impact, and a quantitative study of grain erosion requires accurate data near threshold, where no experimental data are available and where numerical calculations are subject to uncertainties. At high streaming velocities $\left(\Delta v>50 \mathrm{~km} \mathrm{~s}^{-1}\right)$, attained in shocks propagating in the warm component of the medium, sputtering is dominated by $\mathrm{H}$ and He impact, as shown by Draine \& Salpeter (1979b) and Tielens et al. (1994).

\section{Concluding remarks}

A quantitative study of sputtering processes in C-shocks, with velocities up to about $50 \mathrm{~km} \mathrm{~s}^{-1}$, requires data which are accurate near threshold, in a domain of energy where the sputtering yields have, until recently, been determined by extrapolation of experimental measurements at energies above $100 \mathrm{eV}$. In view of the lack of empirical data in the low-energy range, we have computed the sputtering yields for impact by species of various masses on both amorphous carbon and amorphous $\mathrm{SiO}_{2}$ and shown that sputtering by heavy particles dominates at low velocities. However, these calculations have been made with poorly known thermodynamic data (surface binding energy, displacement and bulk binding energy). Furthermore, the refractory grains in the interstellar medium are likely to be olivine $\left((\mathrm{Mg}, \mathrm{Fe}) \mathrm{SiO}_{4}\right)$, for which no experimental or theoretical data currently exist. Accordingly, we are presently calculating the thermodynamic data required to determine the sputtering yields of $\mathrm{Si}, \mathrm{O}, \mathrm{Mg}$ and $\mathrm{Fe}$ in olivine (each species has its 
own displacement and binding energies), and these results will be reported in a future publication.

The release of silicon into the gas phase is followed by the formation of $\mathrm{SiO}$ whose emission is a known signature of molecular outflows. The computed $\mathrm{SiO}$ column densities and line profiles are found to be comparable with those observed (Schilke et al. 1997). SiO reacts with $\mathrm{OH}$ in the postshock gas, forming $\mathrm{SiO}_{2}$; this may explain the low upper limits to the fractional abundance of $\mathrm{SiO}$ deduced from observations of dense dark clouds. It should be noted that $\mathrm{Si}\left(\mathrm{O}_{2}, \mathrm{O}\right) \mathrm{SiO}$ is a key reaction for the production of $\mathrm{SiO}$ in dense clouds, and it would be interesting to measure the rate coefficient for this neutral-neutral reaction below $300 \mathrm{~K}$.

The present study has been limited to $\mathrm{SiO}$ and silicon-bearing species. However, the abundances of ammonia and methanol are also enhanced in some molecular outflows (Bachiller et al. 1993, 1995). These molecules are believed to be the products of desorption in shocks, although it is not clear whether they are released directly from the grain mantles or form in the gas phase from simpler molecules released from the grains. Finally, we note that $\mathrm{H}_{2}$ infrared emission has been detected in a number of outflows and is also considered to be a tracer of shocks (Davis \& Eisloeffel 1995). It is our intention to include in our code the processes of excitation of these molecules and to calculate their line intensities and profiles in order to further constrain the models.

\section{Acknowledgements}

We thank David Field, Paul May, Peter Schilke and Malcolm Walmsley for their collaboration in this work which has been partially supported by both the "Alliance" and the "Procope" science programmes. We are grateful to Matthieu Kopp for his help in preparing the manuscript.

\section{References}

Anders E., Grevesse N., 1989, Geochim. Cosmochim. Acta, 53, 197

Andersen H.H. and Bay H.L., 1981, in Sputtering by Particle Bombardment I, ed. R. Behrisch (Springer-Verlag, New York), p.145

Bachiller R., Martín-Pintado J., Fuente A., 1991, A\&A, 243, L21

Bachiller R., Martín-Pintado J., Fuente A., 1993, ApJ, 417, L45

Bachiller R., Liechti S., Walmsley C. M., Colomer F., 1995, A\&A, 295, L51

Barlow M.J., 1978, MNRAS, 183, 367

Betz G., Wehner G.K., 1983, in Sputtering by Particle Bombardment II, ed. R. Behrisch (Springer-Verlag, New York), p.11

Davis C. J., Eisloeffel J., 1995, A\&A, 300, 851

Draine B.T., 1980, ApJ, 241, 1021

Draine B.T., 1990, in The Evolution of the Interstellar Medium, ed. Blitz, ASP Conf. Series, 12, 193

Draine B.T., 1995, Ap. Sp. Sci., 233, 111 
Draine B.T., Roberge W.G., Dalgarno A., 1983, ApJ, 264, 485

Draine B.T., Salpeter E.E., 1979a, ApJ, 231, 77

Draine B.T., Salpeter E.E., 1979b, ApJ, 231, 438

Field D., May P.W., Pineau des Forêts G., Flower D.R., 1996, MNRAS, 280, 447

Flower D.R., Pineau des Forêts G., 1994, MNRAS, 268, 724

Flower D.R., Pineau des Forêts G., 1995, MNRAS, 275, 1049

Flower D.R., Pineau des Forêts G., Walmsley C.M., 1995, A\&A, 294, 815

Gry C., Boulanger F., Falgarone E., Pineau des Forêts G., Lequeux J., 1997, submitted to $\mathrm{A} \& \mathrm{~A}$

Havnes O., Hartquist T.W., Pillip W., 1987, in Physical Processes in Interstellar Clouds, G.E. Morfill and M. Scholer eds (Reidel, Dordrecht), p. 389

Heck L., Flower D.R., Pineau des Forêts G., 1990, Computer Phys. Comm., 58, 169

Herbst E., Millar T.J., Wlodek S., Bohme D.K., 1989, A\&A, 222, 205

Jansen D.J., Spaans M., Hogerheijde M.R., van Dishoeck E.F., 1995, A\&A, 303, 541

Jones A.P., Tielens A.G.G.M., Hollenbach D.J., McKee C.F., 1994, ApJ. 433, 797

Jones A.P., Tielens A.G.G.M., Hollenbach D.J., 1996, ApJ., in press

Langer W.D., Glassgold A.E., 1990, ApJ, 352, 123

Martin-Pintado J., Bachiller R., Fuente A., 1992, A\&A, 254, 315

Mathis J.S., Rumpl W., Nordsieck K.H., 1977, ApJ, 217, 425

McKee C.F., 1989, in Interstellar Dust, ed. L.J. Allamandola and A.G.G.M. Tielens (Dordrecht, Kluwer), p.431

McKee C.F., Hollenbach D.J., Seab C.G., Tielens A.G.G.M., 1987, ApJ. 318, 674

Mullan D.J., 1971, MNRAS, 153, 145

Raga A.C., Cabrit S., Cantó J., 1995, MNRAS, 273, 422

Roth J., Bohdansky J., Ottenberger W., 1979, Rpt. IPP 9/26, Max-Planck-Institut fur Plasmaphysik, Garching

Schilke P., Walmsley C.M., Pineau des Forêts G., Flower D.R., 1997, A\&A, in press

Seab C.G., 1987, in Interstellar Processes, D.J. Hollenbach and H.A.Thronson eds (Reidel, Dordrecht), p. 491

Seab C.G., Shull J.M., 1983, ApJ. 115, 227

Smith M.D., 1991, MNRAS, 253, 175

Sofia U.J., Cardelli J.A., Savage B.D., 1994, ApJ, 430, 650

Sofia U.J., Savage B.D., Cardelli J.A., 1993, ApJ, 423, 251

Spitzer L. Jr., 1976, Comments Astrophys. 6, 177

Tielens A.G.G.M., McKee C.F., Seab C.G., Hollenbach D.J., 1994, ApJ. 431, 321

Turner B.E., 1991, ApJ, 376, 573

van Dishoeck E.F., Jansen D.J., Phillips T.G., 1993, A\&A, 279, 541

Zhang Q., Ho P.T.P., Wright M.C.H., Wilner D.J., 1995, ApJ, 451, L71

Ziegler J.F., Biersack J.P., Littmark U., 1985, The Stopping Range of Ions in Solids, vol. 1, Ziegler J.F., ed, (Pergamon, New York)

Ziurys L.M., Friberg P., Irvine W.M., 1989, ApJ, 343, 201 


\section{Discussion}

Greenberg: Are there ever pure silicate cores as a major dust constituent? According to my model the silicate cores are protected by organic refractory mantles so that the observed $\mathrm{SiO}$ in shocks comes from these mantles, where it has been trapped during the molecular cloud phase.

Pineau des Forêts: In these initial calculations, we have adopted a very simple grain model consisting of an icy mantle and a refractory core.

Avery: Sputtering is a rather energetic process. Could you comment on whether it is likely to liberate entire molecules from grains or will it dissociate them? And if sputtering dissociates the molecules, what other shock processes are gentle enough to explain the liberation of entire molecules such as $\mathrm{CH}_{3} \mathrm{OH}$ and $\mathrm{H}_{2} \mathrm{CO}$ which seems necessary to explain observed enhancements in outflows?

Pineau des Forêts: There is a profile of ion-neutral velocity differences within the shock. The grain mantles are completely removed at low relative velocities, and the refractory cores are then sputtered at higher velocities. It is possible, and assumed in the model, that saturated molecules return to the gas phase from the mantles.

Tielens: Silicate sputtering will result in $\mathrm{SiO}, \mathrm{SiO}_{2}$ and $\mathrm{Si}_{2}$ ejection, besides $\mathrm{Si}$ and $\mathrm{O}$.

Pineau des Forêts: TRIM incorporates the interactions between the incident and the target atoms individually, and the ejection of molecular fragments is not taken into account.

Tielens: There are ample data on near-threshold sputtering for metals (but not for astrophysically important compounds such as silicate and graphite) which are well fitted by our semi-empirical fits. Comparing your theoretical models to our semiempirial formalism, I would conclude that you severely overestimate the surface binding energy (which is not equal to the bulk heat of formation).

Pineau des Forêts: No comment.

Tielens: At low velocities, sputtering is by the heaviest species (C, N and O) which collide at $E_{k} \sim 500-1000 \mathrm{eV}$. So, the uncertainty associated with the threshold energy is not too serious.

Pineau des Forêts: In the shock models, the impact velocities are of the order of $30 \mathrm{~km} / \mathrm{s}$ corresponding to impact energies less than $100 \mathrm{eV}$ for $\mathrm{C}, \mathrm{N}$ and $\mathrm{O}$. In this case, the question of the threshold energy becomes more significant. 


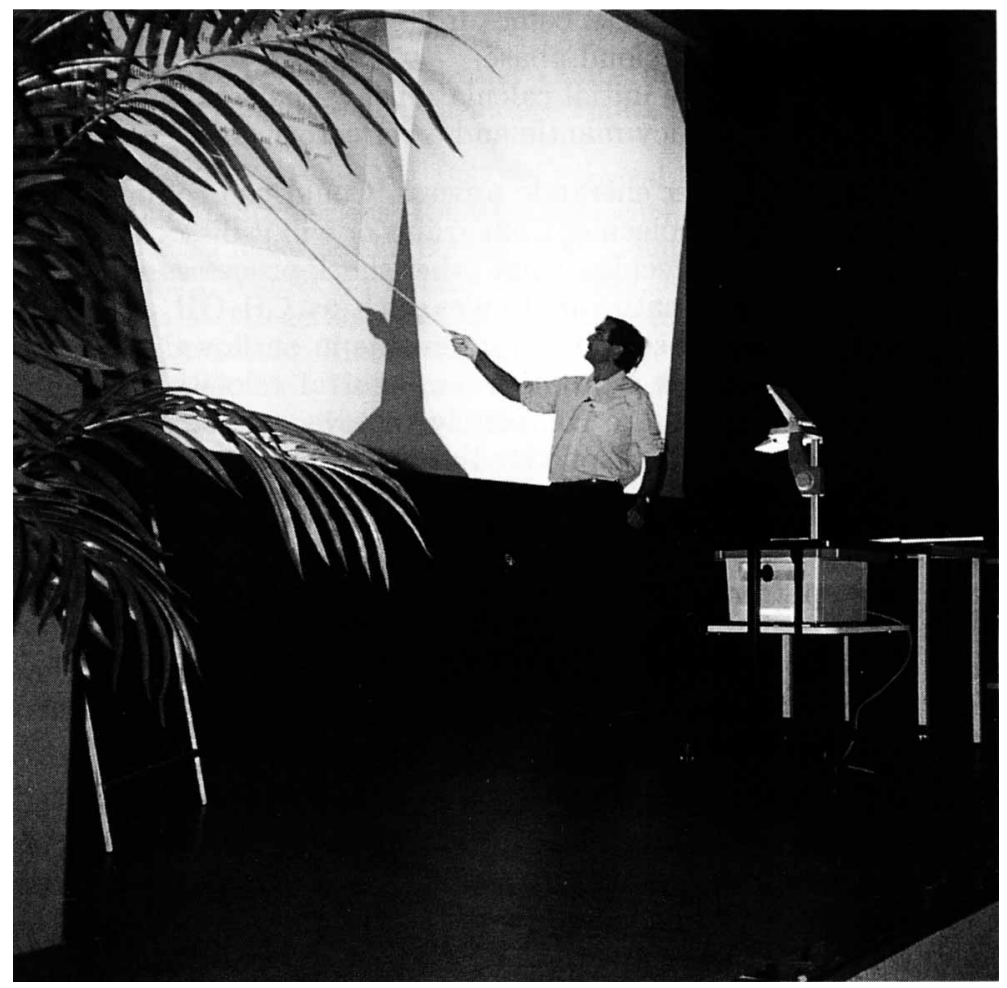

